\title{
ISO 17025 Accreditation for the Bioforensic Light and Electron Microscopy Imaging of Castor Bean Products and the Immuno Electron Microscopy Identification of Ricin in Castor Bean Products
}

\author{
Robert Pope, Brian Leroux, Henry Lupari and Mary Beth Friss \\ BNBI/NBACC/DHS, Fort Detrick, Maryland, United States
}

Ricinus communis, commonly known as castor bean, is an ornamental species of plant that produces castor beans, and is commercially grown for the production of castor oil (chiefly ricinoleic acid). One of the byproducts of castor oil production is the protein toxin ricin, which is contained within castor beans (Figure 1A). Analysis of castor bean products and identification of ricin in castor bean samples was investigated using light microscopy, scanning electron microscopy, transmission electron microscopy, and immuno electron microscopy. These different methods of microscopy all demonstrate some level of applicability in the identification of ricin in castor bean samples. For this experiment, castor beans were ground, inactivated with either traditional electron microscopy (EM) fixative (4\% paraformaldehyde with $1 \%$ glutaraldehyde), or immuno EM fixative (1\% paraformaldehyde with $0.1 \%$ glutaraldehyde), and imaged via light and electron microscopy. Samples of ground castor beans imaged using stereo microscopy clearly demonstrate brown to tan shell fragments, whitish seed endosperm, and abundant glossy castor oil (Figure 1B). Higher magnification imaging of epoxy-embedded, sectioned, and toluidine blue-stained ground castor bean samples show the presence of cell wall material (dark blue), castor oil droplets (dark tan), and aleurone grains (light blue) that are characteristic of castor beans (Figure 1C). Scanning electron microscopy analysis demonstrate cell wall material (green), with castor oil droplets (yellow), and crystals of ricin protein (red, Figure 1D). Transmission electron microscopy analysis shows an aleurone grain that is characteristic of castor beans, with a protein rich crystalloid, surrounded by a proteinaceous material, and containing a lipid body in a globoid space (Figure 1E) [1]. Immuno electron microscopy using anti-ricin A chain antibodies [2, 3] (Mab Ric-03-A-G1) conjugated to $40 \mathrm{~nm}$ gold spheres demonstrates that the crystalline structure inside the aleurone grain is ricin protein (Figure 1F). Additional immuno electron microscopy using anti-ricin B chain antibodies demonstrates similar staining (data not shown).

ISO 17025 accreditation of these methods for the analysis of castor bean products, and for the identification of ricin in castor bean products required the validation of the procedures, the competency testing of personnel on these methods, and the proficiency testing of the laboratory to ensure the methods were accurate, reliable, and repeatable. For all processes, samples of castor bean mash were blinded with other products, such as kidney beans (Phaseolus vulgaris), soybeans (Glycine max), and rosary peas (Abrus precatorius). Individuals had to identify the specific type of sample, and in the case of immuno $\mathrm{EM}$, had to identify the structures containing ricin protein. Also, during the ISO 17025 accreditation process, equipment, reagents and training records were tracked, standards were used to verify that the LM, SEM and TEM were producing the correct magnification, and that the software used for capturing images produced the correct magnification [4]. 


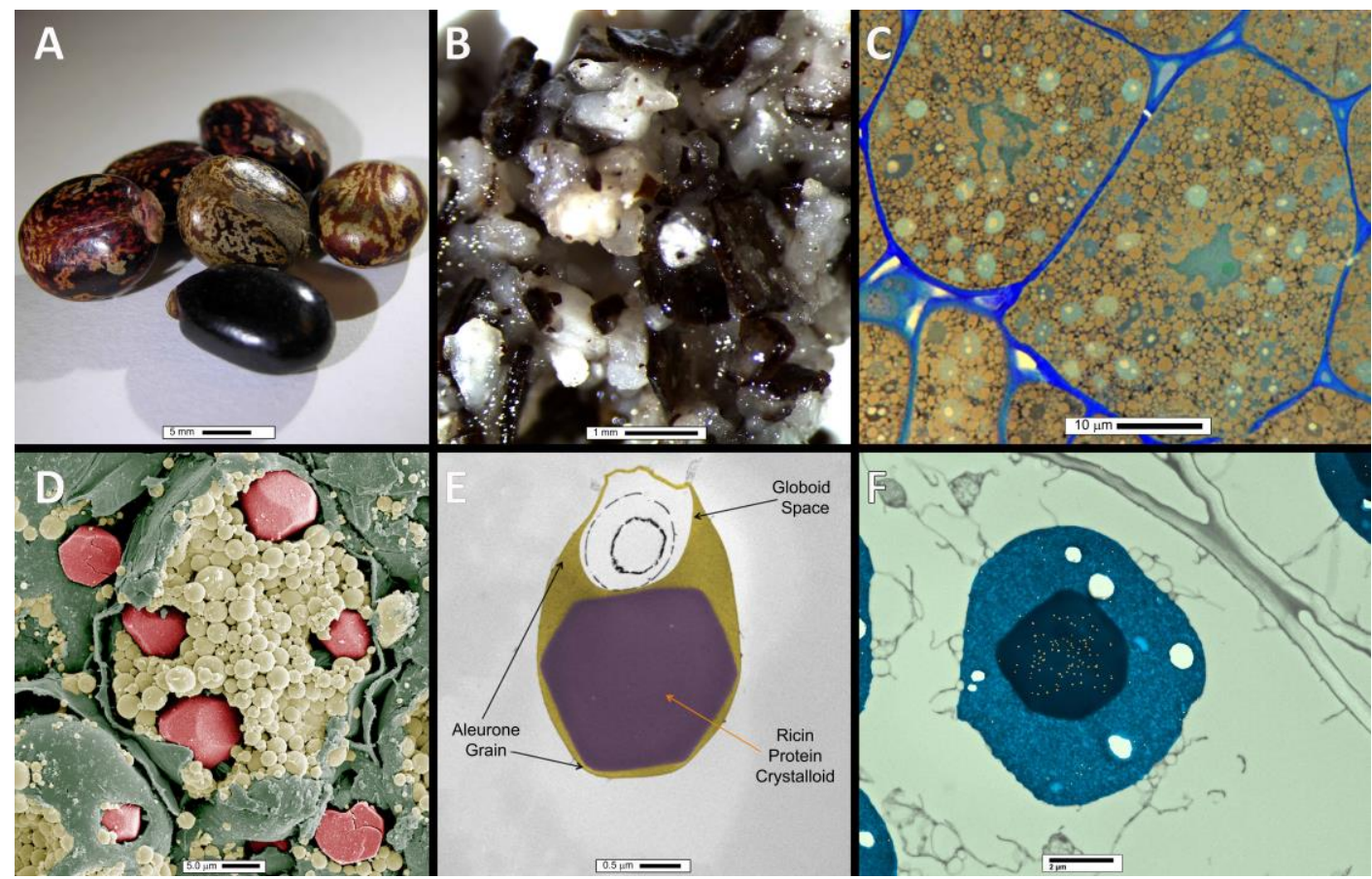

Figure 1. FIG. 1. A) Digital image of castor beans (Ricinus communis). B) Stereo microscopy image of ground castor beans. C) Light microscopy image of 200nm section of castor bean stained with toluidine blue showing cellulose wall in dark blue, castor oil as dark tan droplets, and light blue spherical aleurone grains containing a globoid space with lipid bodies. D) Scanning electron microscopy image of broken open castor bean showing cell wall material in green, castor oil droplets in yellow, and crystals of ricin protein in red. E) Transmission electron microscopy image of castor bean aleurone grain showing ricin protein crystalloid, proteinaceous matrix in yellow, and globoid space with lipid body. F) Immuno EM with anti-ricin A chain antibodies conjugated to $40 \mathrm{~nm}$ gold particles showing ricin crystal in dark blue surrounded by a proteinaceous matrix containing ricin protein. Panels D-F were pseudo colored using Adobe Photoshop.

\section{References}

[1] Tully, RE and Beevers, H (1976) Protein Bodies of Castor Bean Endosperm: Isolation, Fractionation, and the Characterization of Protein Components. Plant Physiology. 58:710-716.

[2] Baldoni, AB et al. (2010) Immunolocalization of ricin accumulation during castor bean (Ricinus communis L.) seed development. International Journal of Plant Biology. 1(12):61-65.

[3] Anderson, GP et al. (2007) Multiplexed fluid array screening of phage displayed anti-ricin single domain antibodies for rapid assessment of specificity, Biotechniques Research Reports. 43(6):806-811.

[4] "This work was funded under Agreement No. HSHQDC-15-C-00064 awarded to Battelle National Biodefense Institute by the Department of Homeland Security Science and Technology Directorate (DHS S\&T) for the management and operation of the National Biodefense Analysis and Countermeasures Center a Federally Funded Research and Development Center. The views and conclusions contained in this document are those of the authors and should not be interpreted as necessarily representing the official policies, either expressed or implied, of the U.S. Department of Homeland Security or the U.S. Government. The Department of Homeland Security does not endorse any products or commercial services mentioned in this presentation. In no event shall the DHS, BNBI or NBACC have any responsibility or liability for any use, misuse, inability to use, or reliance upon the information contained herein. In addition, no warranty of fitness for a particular purpose, merchantability, accuracy or adequacy is provided regarding the contents of this document." All research was conducted following appropriate Federal guidelines (no humans or animals were used in this research). 